\title{
PARTIAL REGULARITY AND EVERYWHERE CONTINUITY FOR A MODEL PROBLEM FROM NON-LINEAR ELASTICITY
}

\author{
NICOLA FUSCO and JOHN E. HUTCHINSON
}

(Received 15 August 1991; revised 4 May 1992)

Communicated by E. N. Dancer

\begin{abstract}
We prove a new energy or Caccioppoli type estimate for minimisers of the model functional $\int_{\Omega}|D \boldsymbol{u}|^{2}+$ (det $D u)^{2}$, where $\Omega \subset \mathbb{R}^{2}$ and $u: \Omega \rightarrow \mathbb{R}^{2}$. We apply this to establish $C^{\infty}$ regularity for minimisers except on a closed set of measure zero. We also prove a maximum principle and use this to establish everywhere continuity of minimisers.
\end{abstract}

1991 Mathematics subject classification (Amer. Math. Soc.): 35J50, 35J60, 73C50.

Keywords and phrases: nonlinear elasticity, partial regularity, elliptic systems, polyconvex, quasiconvex, maximum principle.

\section{Introduction}

Since the first paper by Evans [4] on the subject appeared, partial regularity of minimisers (that is, smoothness except on a closed set of measure zero) of functionals of the form

$$
I[u]=\int_{\Omega} F(D u) d x
$$

where $\Omega \subset \mathbb{R}^{n}, u: \Omega \rightarrow \mathbb{R}^{N}$ and $F$ is quasi-convex, has been extensively studied (see Acerbi and Fusco [1], Evans and Gariepy [5], Fusco and Hutchinson [6], Giaquinta and Modica [9]).

Recently in [7] the authors have studied the partial regularity of minimisers of certain polyconvex functionals (that is functionals which are convex in the various minors of the matrix $[D u]$ ). Polyconvex functionals are quasiconvex and arise in

(C) 1994 Australian Mathematical Society 0263-6115/94 \$A2.00+0.00 
non-linear elasticity (Ball $[2,3])$, and all known 'natural' examples of quasiconvex functionals are polyconvex.

A model problem covered by the results in Fusco and Hutchinson [7] is given by $N=n=2$ and

$$
I[u]=\int_{\Omega}|D u|^{2}+(\operatorname{det} D u)^{2} .
$$

The first term in the integrand is the usual Dirichlet energy (for line elements); the second term is the Dirichlet energy for area elements (compare Fusco and Hutchinson [7]). There is clearly no loss of generality in having the same coefficient in front of each term, as follows by a scaling argument.

We show in particular that mininimisers of this functional are smooth except on a closed set of measure zero, and are everywhere continuous.

Although simple, this example already contains all the difficulties which appear in handling the general case to which the main theorem in Fusco and Hutchinson [7] applies. Firstly, the set of competing functions, those for which the integral is finite, is not a linear subspace of $W^{1,2}$. Secondly, the term $(\operatorname{det} D u)^{2}$ can grow, in certain directions, like a fourth power of $D u$, which one has no way of controlling by using the leading term $|D u|^{2}$ appearing in $I[u]$.

These two difficulties were overcome in Fusco and Hutchinson [7]. There we used the standard technique of blowing up a minimiser $u$ of $I$ around a convergent sequence of points in order to obtain a sequence $v_{m}$ of functions which converges weakly in $W^{1,2}$ to the solution $v$ of a linear elliptic system with constant coefficients. Then the crucial fact that we proved in order to obtain the decay Lemma 3.1 and hence the partial regularity result Theorem 3.2 was that the functions $v_{m}$ converge strongly in $W^{1,2}$ to $v$.

The first main result in this paper is an energy estimate of Caccioppoli type which leads to an alternative proof of the decay estimate Lemma 3.1. Namely, we prove that if $u \in W^{1,2}$ is a minimizer of $I$ then for any ball $B_{R} \subset \subset \Omega$

$$
\int_{B_{R} / 2}|D u|^{2}+(\operatorname{det} D u)^{2} \leq \frac{c}{R^{2}} \int_{B_{R}}\left|u-u_{R}\right|^{2}+\frac{c}{R^{2}}\left(\int_{B_{R}}|D u|^{2}\right)^{2} .
$$

This estimate is proved using the comparison function (2.9) introduced in Fusco and Hutchinson [7]. Once one has (1.3), the proof of the Lemma 3.1 becomes simpler than in [7].

In order to make clear the ideas and techniques used in the proof of partial regularity, we restrict ourselves to the model case $I[u]$. To prove Theorem 3.2 in the general case one should use the appropriate version of the estimate (1.3) containing all the higher order minors of the matrix $[D u]$. This requires only technical complications, but no new ideas. 
Finally, we remark that the blow-up argument used here and in [7] works because the leading term in $I[u]$ is quadratic and there is no degeneracy in ellipticity. It would not work if we had something like

$$
I[u]=\int_{\Omega}|D u|^{p}+(\operatorname{det} D u)^{p},
$$

with $p>2$, or its $n$-dimensional version. However, the extension of Theorem 3.2 to functionals having a degenerate elliptic leading term will be treated in a forthcoming paper by the two authors.

The second main result in this paper is a proof of everywhere continuity of minimisers of the functional $I[u]$. This uses an earlier unpublished maximum principle result of the authors, and of Leonetti $[10,11]$. We give here a simple geometric proof. Everywhere continuity follows from an application of the Courant-Lebesgue lemma. We remark that this result does not extend to the general class of problems considered in [7]. The second author would like to thank Michael Grüter and Ulrich Dierkes for pointing out the relevance of the Courant-Lebesgue lemma in this setting.

\section{The Energy Estimate}

If $\Omega \subset \mathbb{R}^{2}$ is a bounded open set and $\lambda$ is any real number, we consider for $v \in W^{1.2}\left(\Omega ; \mathbb{R}^{2}\right)$ the functional

$$
I_{\lambda}[v, \Omega]=\int_{\Omega}|D v|^{2}+\lambda^{2}(\operatorname{det} D v)^{2} .
$$

If $Q \geq 1$ is a real number we say that $u \in W^{1,2}\left(\Omega ; \mathbb{R}^{2}\right)$ is a $Q$-minimiser of $I_{\lambda}$ if, for any $\phi \in W^{1,2}\left(\Omega ; \mathbb{R}^{2}\right)$ whose support is a compact subset of $\Omega$,

$$
I_{\lambda}(u) \leq Q I_{\lambda}(u+\phi) .
$$

If $Q=1$ then $u$ is a minimiser.

In the following we write

$$
f_{E} g=\frac{1}{|E|} \int_{E} g=g_{E}
$$

for any measurable set $E$ of positive measure and any $g \in L^{1}\left(E ; \mathbb{R}^{k}\right)$. If $E$ is the open ball $B_{R}\left(x_{0}\right)$ we also write

$$
f_{B_{R}\left(x_{0}\right)} g=(g)_{x_{0}, R}=g_{R} .
$$

The key ingredient in obtaining the decay Lemma 3.1 is the following energy estimate for a $Q$-minimiser of $I_{\lambda}$. 
LEMMA 2.1. If $u \in W^{1,2}\left(\Omega ; \mathbb{R}^{2}\right)$ is a $Q$-minimiser of $I_{\lambda}$, then there exists a constant $c$ depending only on $Q$ such that for any $B_{R} \subset \subset \Omega$

$$
\int_{B_{R / 2}}|D u|^{2}+\lambda^{2}(\operatorname{det} D u)^{2} \leq \frac{c}{R^{2}} \int_{B_{R}}\left|u-u_{R}\right|^{2}+\frac{c \lambda^{2}}{R^{2}}\left(\int_{B_{R}}|D u|^{2}\right)^{2} .
$$

PROOF. Fix $B_{R} \subset \subset \Omega$ and $R / 2<s<t<R$. Define

$$
E_{s, t}=\left\{\rho \in(s, t): \int_{\partial B_{\rho}}|D u|^{2} \leq \frac{2}{t-s} \int_{B_{t} \backslash B_{s}}|D u|^{2}\right\} .
$$

Then

$$
\left|E_{s, t}\right| \geq \frac{t-s}{2} .
$$

Let $\omega=x /|x|$. For a.e. $\rho \in(s, t)$ the function $\omega \mapsto u(\rho \omega)$ belongs to $W^{1,2}\left(\partial B_{1}\right)$ and

$$
\left|u(\rho \omega)-u_{\partial B_{\rho}}\right| \leq c \sqrt{\rho}\left(\int_{\partial B_{\rho}}|D u|^{2}\right)^{1 / 2}
$$

for all $\omega$ (taking the continuous representative of $\omega \mapsto u(\rho \omega)$ ), where $u_{\partial B_{\rho}}=$ $1 /(2 \pi \rho) \int_{\partial B_{\rho}} u$.

For each $\rho$ we introduce the comparison function

$$
\phi(r \omega)= \begin{cases}u_{\partial B_{\rho}} & \text { if } r \leq s \\ \frac{\rho-r}{\rho-s} u_{\partial B_{\rho}}+\frac{r-s}{\rho-s} u(\rho \omega) & \text { if } s \leq r \leq \rho \\ u(r \omega) & \text { if } \rho \leq r \leq R .\end{cases}
$$

It easily follows that

$$
|D \phi(r \omega)| \leq c\left(\frac{\left|u(\rho \omega)-u_{\partial B_{\rho}}\right|}{\rho-s}+|D u(\rho \omega)|\right)
$$

and

$$
|\operatorname{det} D \phi(r \omega)| \leq c \frac{\left|u(\rho \omega)-u_{\partial B_{\rho}}\right|}{\rho-s}|D u(\rho \omega)|
$$

for any $r \in[s, \rho]$ (recall $r / 2<s<\rho<R$ ).

From (2.8), (2.10), (2.11) and the $Q$-minimising property of $u$, it follows that for a.e. $\rho \in E_{s, t}$

$$
\begin{aligned}
\int_{B_{\rho}}|D u|^{2} & +\lambda^{2}(\operatorname{det} D u)^{2} \leq Q \int_{B_{\rho} \backslash B_{s}}|D \phi|^{2}+\lambda^{2}(\operatorname{det} D \phi)^{2} \\
& \leq \frac{c}{\rho-s} \int_{\partial B_{\rho}}\left|u-u_{\partial B_{\rho}}\right|^{2}+c(\rho-s) \int_{\partial B_{\rho}}|D u|^{2}+\frac{c \lambda^{2}}{\rho-s} \int_{\partial B_{\rho}}\left|u-u_{\partial B_{\rho}}\right|^{2}|D u|^{2} \\
(2.12) & \leq \frac{c}{\rho-s} \int_{\partial B_{\rho}}\left|u-u_{R}\right|^{2}+c(\rho-s) \int_{\partial B_{\rho}}|D u|^{2}+\frac{c \rho \lambda^{2}}{\rho-s}\left(\int_{\partial B_{\rho}}|D u|^{2}\right)^{2} .
\end{aligned}
$$


From (2.7) it follows that

$$
\left|\left\{\rho \in E_{s, t}: \rho-s \geq(t-s) / 4\right\}\right| \geq(t-s) / 4 .
$$

Multiplying (2.12) by $\rho-s$ and integrating with respect to $\rho$ over $E_{s, t}$, and again using (2.7) and the definition of $E_{s, t}$, we see that

$$
\frac{(t-s)^{2}}{16} \int_{B_{s}}|D u|^{2}+\lambda^{2}(\operatorname{det} D u)^{2}
$$

$$
\leq c \int_{B_{i} \backslash B_{s}}\left|u-u_{R}\right|^{2}+c(t-s)^{2} \int_{B_{t} \backslash B_{s}}|D u|^{2}+\frac{c R \lambda^{2}}{t-s}\left(\int_{B_{i} \backslash B_{s}}|D u|^{2}\right)^{2} .
$$

Dividing through by $(t-s)^{2}$ and using the 'hole-filling' trick, (that is adding $c \int_{B_{s}}|D u|^{2}$ $+c \int_{B_{s}} \lambda^{2}(\operatorname{det} D u)^{2}$ to both sides of the inequality) it follows there exists $\theta \in(0,1)$ such that

$$
\begin{aligned}
I_{\lambda}\left(u ; B_{s}\right) \leq \theta I_{\lambda}\left(u ; B_{t}\right)+\frac{c}{(t-s)^{2}} \int_{B_{R} \backslash B_{R / 2}} & \left|u-u_{R}\right|^{2} \\
& +\frac{c R \lambda^{2}}{(t-s)^{3}}\left(\int_{B_{R} \backslash B_{R / 2}}|D u|^{2}\right)^{2} .
\end{aligned}
$$

The Lemma now follows from a straightforward extension of Giaquinta [8, Chapter 5, Lemma 3.1].

\section{Partial Regularity}

The essential tool in obtaining the partial regularity result on Theorem 3.2 is the following decay estimate for the quantity

$$
U(x, r)=f_{B_{r}(x)}\left|D u-(D u)_{r}\right|^{2}+\left(\operatorname{det}\left(D u-(D u)_{r}\right)\right)^{2} .
$$

LEMMA 3.1. Let $u \in W^{1,2}\left(\Omega ; \mathbb{R}^{2}\right)$ be a minimiser of the functional I[.]. Suppose $M>0$. Then for some constant $c(M)$ and any $\tau \in(0,1 / 2)$ there exists $\epsilon(\tau, M)$ such that if

$$
\left|(D u)_{x, r}\right| \leq M \quad \text { and } \quad U(x, r)<\epsilon
$$

then

$$
U(x, \tau r) \leq c(M) \tau^{2} U(x, r) .
$$


PROOF. Fix $M$. Arguing by way of contradiction we assume that there exists a sequence of balls $B_{r_{m}} \subset \subset \Omega$ for which

$$
\left|(D u)_{x_{m}, r_{m}}\right| \leq M \quad \text { and } \quad \lambda_{m}^{2}=U\left(x_{m}, r_{m}\right) \rightarrow 0
$$

but

$$
\frac{U\left(x_{m}, \tau r_{m}\right)}{\lambda_{m}^{2}}>c(M) \tau^{2},
$$

where $c(M)$ will be chosen later.

We set $A_{m}=(D u)_{x_{m}, r_{m}}$ and

$$
v_{m}(y)=\frac{u\left(x_{m}+r_{m} y\right)-(u)_{x_{m}, r_{m}}-r_{m} A_{m} y}{\lambda_{m} r_{m}}
$$

for all $y \in B_{1}(0)$.

Then

$$
\int_{B_{1}}\left|D v_{m}\right|^{2}+\lambda_{m}^{2}\left(\operatorname{det} D v_{m}\right)^{2}=1
$$

and $\left(v_{m}\right)_{0,1}=0$. So we may suppose on passing to a subsequence that

$$
\begin{array}{rlr}
D v_{m} & \rightarrow D v & \text { weakly in } L^{2}\left(B_{1}\right), \\
v_{m} & \rightarrow v \quad \text { strongly in } L^{2}\left(B_{1}\right), \\
\lambda_{m} \operatorname{det} D v_{m} & \rightarrow 0 \quad \text { weakly in } L^{2}\left(B_{1}\right), \\
A_{m} & \rightarrow A . &
\end{array}
$$

The third claim in (3.8) comes from observing that $\lambda_{m}$ det $D v_{m}$ converges weakly in $L^{2}\left(B_{1}\right)$, but also for any $\phi \in C_{0}^{1}\left(B_{1}\right)$

$$
\int \operatorname{det} D v_{m} \phi \rightarrow \int \operatorname{det} D v \phi,
$$

as one can check by writing det $D v_{m}$ in divergence form.

It is also immediate to check that $v_{m}$ minimises the functional

$$
w \mapsto \int_{B_{1}} \lambda_{m}^{2}|D w|^{2}+\left(\operatorname{det}\left(\lambda_{m} D w+A_{m}\right)\right)^{2} .
$$

We introduce the bilinear form $\odot$ defined by

$$
\operatorname{det}(A+B)=\operatorname{det} A+A \odot B+\operatorname{det} B
$$

where $A, B \in \operatorname{Hom}\left(\mathbb{R}^{2}, \mathbb{R}^{2}\right)$. 
Expanding out the second term on the right side of (3.10) and expressing det $\left(\lambda_{m} D w\right.$ $+A_{m}$ ) in divergence form, it follows that $v_{m}$ minimises the functional

$$
w \mapsto \int_{B_{1}}|D w|^{2}+\left(A_{m} \odot D w+\lambda_{m} \operatorname{det} D w\right)^{2} .
$$

Hence $v_{m}$ satisfies the Euler-Lagrange system

$$
0=\int_{B_{1}} D v_{m} D \phi+\left(A_{m} \odot D v_{m}+\lambda_{m} \operatorname{det} D v_{m}\right)\left(A_{m} \odot D \phi+\lambda_{m} D v_{m} \odot D \phi\right)
$$

for all $\phi \in W_{0}^{1,2}\left(B_{1} ; \mathbb{R}^{2}\right)$. Letting $m \rightarrow \infty$ and using (3.8) we obtain

$$
0=\int D v D \phi+(A \odot D v)(A \odot D \phi) .
$$

Thus $v$ satisfies a linear elliptic system with constant coefficients.

By standard regularity results (see for example Giaquinta [8, Chapter III]) we have for any $\tau \in(0,1)$ that

$$
f_{B_{\tau}}\left|D v-(D v)_{\tau}\right|^{2} \leq c \tau^{2} f_{B_{1}}\left|D v-(D v)_{\tau}\right|^{2} \leq c \tau^{2}
$$

and

$$
\left|(D v)_{2 \tau}-(D v)_{\tau}\right|^{2} \leq c \tau^{2}
$$

where $c$ depends only on the ellipticity constants of the system (3.14) and hence only on $M$. Notice that the last inequality in each case follows from (3.7) and (3.8).

On rescaling (3.1) we have for any $\tau \in(0,1 / 2)$ that

$$
\frac{U\left(x_{m}, \tau r_{m}\right)}{\lambda_{m}^{2}}=f_{B_{\mathrm{r}}}\left|D v_{m}-\left(D v_{m}\right)_{\tau}\right|^{2}+\lambda_{m}^{2}\left(\operatorname{det}\left(D v_{m}-\left(D v_{m}\right)_{\tau}\right)\right)^{2}
$$

Setting

$$
w_{m}(y)=v_{m}(y)-\left(D v_{m}\right)_{\tau} y-\left(v_{m}\right)_{2 \tau}
$$

and using both the fact that $v_{m}$ minimises the functional (3.12) and the divergence structure of 'det', we see that $w_{m}$ minimises the functional

$$
w \mapsto \int_{B_{1}}|D w|^{2}+\left(\left(A_{m}+\lambda_{m}\left(D v_{m}\right)_{\tau}\right) \odot D w+\lambda_{m} \operatorname{det} D w\right)^{2} .
$$

Hence $w_{m}$ is a $Q$-minimiser of $I \lambda_{m}\left[w ; B_{1}\right]$ for some $Q=Q(M)$. 
Then from Lemma 2.1 we obtain for $\tau \in(0,1 / 2)$ that

$$
\begin{aligned}
\frac{U\left(x_{m}, \tau r_{m}\right)}{\lambda_{m}^{2}} & =f_{B_{\tau}}\left|D w_{m}\right|^{2}+\lambda_{m}^{2}\left(\operatorname{det} D w_{m}\right)^{2} \\
& \leq \frac{c}{\tau^{2}} \int_{B_{2 \tau}}\left|w_{m}-\left(w_{m}\right)_{2 \tau}\right|^{2}+c \lambda_{m}^{2}\left(f_{B_{2 \tau}}\left|D w_{m}\right|^{2}\right)^{2} .
\end{aligned}
$$

Passing to the limit as $m \rightarrow \infty$ and using (3.8), the Poincaré inequality, (3.15) and (3.16), we obtain for $\tau \in(0,1 / 2)$ that

$$
\begin{aligned}
\limsup _{m \rightarrow \infty} \frac{U\left(x_{m}, \tau r_{m}\right)}{\lambda_{m}^{2}} & \leq \frac{c}{\tau^{2}} f_{B_{2 \tau}}\left|v-(v)_{2 \tau}-(D v)_{\tau} y\right|^{2} \\
& \leq \frac{c}{\tau^{2}} f_{B_{2 \tau}}\left|v-(v)_{2 \tau}-(D v)_{2 \tau} y\right|^{2}+\left|(D v)_{2 \tau}-(D v)_{\tau}\right|^{2} \\
& \leq c\left(f_{B_{2 \tau}}\left|D v-(D v)_{2 \tau}\right|^{2}\right)+c \tau^{2} \\
& \leq c_{1}(M) \tau^{2}
\end{aligned}
$$

This contradicts (3.5) if $c(M)$ is chosen larger than $c_{1}(M)$.

A standard iteration and bootstrapping argument ([7, Lemma 6.1] or [8, Chapter VI]) implies:

THEOREM 3.2. Let $u \in W_{l o c}^{1,2}\left(\Omega ; \mathbb{R}^{2}\right)$ be a local minimiser of the functional $I[\cdot]$. Then $u \in C^{1, \infty}\left(\Omega_{0}\right)$, where $\Omega_{0}$ is an open set such that $\left|\Omega \backslash \Omega_{0}\right|=0$. Moreover

$$
\begin{aligned}
\Omega_{0}=\left\{x \in \Omega: \limsup _{r \rightarrow 0}\left|(D u)_{x, r}\right|<\infty,\right. & \lim _{r \rightarrow 0} f_{B_{r}(x)}\left|D u-(D u)_{x, r}\right|^{2}=0, \\
& \text { and } \left.\lim _{r \rightarrow 0} f_{B_{r}(x)}\left(\operatorname{det}\left(D u-(D u)_{x, r}\right)\right)^{2}=0\right\} .
\end{aligned}
$$

\section{Everywhere Continuity}

We first establish a maximum principle using a geometric-type argument. See Leonetti $[10,11]$ for another proof.

Suppose that

$$
u: \Omega\left(\subset \mathbb{R}^{n}\right) \rightarrow \mathbb{R}^{N}
$$

is a minimiser of a functional of the form

$$
I[u]=\int_{\Omega} F\left(|D u|,\left|\Lambda_{2} D u\right|, \ldots,\left|\Lambda_{n} D u\right|\right) .
$$


Here $\left|\Lambda_{k} D u(x)\right|$ is the Euclidean norm of the map

$$
\Lambda_{k} \operatorname{Du}(x): \Lambda_{k} \mathbb{R}^{n} \rightarrow \Lambda_{k} \mathbb{R}^{N},
$$

given by

$$
\Lambda_{k} D u(x)\left(w_{1} \wedge \cdots \wedge w_{k}\right)=D u(x)\left(w_{1}\right) \wedge \cdots \wedge D u(x)\left(w_{k}\right),
$$

and

$$
\left|\Lambda_{k} D u(x)\right|^{2}=\sum_{i_{1}<\cdots<i_{k}}\left|\Lambda_{k} D u(x)\left(\tau_{i_{1}} \wedge \cdots \wedge \tau_{i_{k}}\right)\right|^{2}
$$

for any orthonormal basis $\tau_{1}, \ldots, \tau_{k}$ of $\mathbb{R}^{n}$.

Note that in case $n=N=2$, then det $D u(x)$ can be regarded as the map $\Lambda_{2} D u(x)$ operating on 2-vectors, and $\left|\Lambda_{2} D u(x)\right|^{2}=(\operatorname{det} D u(x))^{2}$.

Assume moreover that $F=F\left(p_{1}, p_{2}, \ldots, p_{n}\right)$ is convex in each of the arguments $p_{k}$ separately, and that $F$ is monotone in the sense that

$$
F\left(q_{1}, q_{2}, \ldots, q_{n}\right)<F\left(p_{1}, p_{2}, \ldots, p_{n}\right)
$$

provided $q_{1}<p_{1}$ and $q_{k} \leq p_{k}$ for $k=2, \ldots, n$. Notice that the model problem (1.1) is of this type.

We then have the following result.

THEOREM 4.1. Let $u \in W^{1, N}\left(\Omega ; \mathbb{R}^{2}\right)$ be a minimiser of the functional $I[\cdot]$, where $I$ is as in (4.2) and is convex and monotone. Let $E \subset \subset \Omega$ be an open set with Lipschitz boundary $\partial E$. Then $u(E) \subset \mathcal{C}(u(\partial E))$, where $\mathcal{C}(u(\partial E))$ is the closed convex hull of $u(\partial E)$.

PROOF. For any closed ball $B_{R}=B_{R}\left(y_{0}\right) \subset \mathbb{R}^{N}$ let $\psi: \mathbb{R}^{N} \rightarrow B_{R}$ be the radial projection map defined by

$$
\psi(y)= \begin{cases}y, & \text { if } y \in B_{R}, \\ y_{0}+R \frac{\left(y-y_{0}\right)}{\left|y-y_{0}\right|}, & y \in \mathbb{R}^{N} \backslash B_{R} .\end{cases}
$$

Fix $y \in \mathbb{R}^{N}$, choose $\tau_{1}=\left(y-y_{0}\right) /\left|y-y_{0}\right|$ and extend $\tau_{1}$ to an orthonormal basis $\tau_{1}, \tau_{2}, \ldots, \tau_{N}$ for $\mathbb{R}^{N}$. Then for any $y \in \mathbb{R}^{N} \backslash B_{R}$

$$
D \psi\left(\tau_{i}\right)=\gamma_{i} \tau_{i}
$$

where $\left|\gamma_{i}\right|<1$. In particular, it follows that

$$
|D \psi(y)(w)|<|w|
$$


for any $0 \neq w \in \mathbb{R}^{N}$.

More generally, it follows from (4.4) and (4.8) that if $y \in \mathbb{R}^{N} \backslash B_{R}$ then

$$
\Lambda_{k} D \psi(y)\left(\tau_{i_{1}} \wedge \cdots \wedge \tau_{i_{k}}\right)=\gamma_{i_{1}} \ldots \gamma_{i_{k}}\left(\tau_{i_{1}} \wedge \cdots \wedge \tau_{i_{k}}\right),
$$

and hence using the orthonormal basis $\tau_{i_{1}} \wedge \cdots \wedge \tau_{i_{k}}$ where $i_{1}<\cdots<i_{k}$, that

$$
\left|\Lambda_{k} D \psi(y)\left(w_{1} \wedge \cdots \wedge w_{k}\right)\right| \leq\left|w_{1} \wedge \cdots \wedge w_{k}\right|
$$

for any $k$-vector $w_{1} \wedge \cdots \wedge w_{k} \in \Lambda_{k} \mathbb{R}^{N}$.

Now suppose $\mathcal{C}(u(\partial E)) \subset B_{R}$ and define

$$
v(x)= \begin{cases}u(x), & \text { if } x \notin E, \\ \psi(u(x)), & \text { if } x \in E\end{cases}
$$

Then $I[u] \leq I[v]$ by the minimising property of $u$.

On the other hand, $\left|\Lambda_{k} D v(x)\right|=\left|\Lambda_{k} D \psi(u(x)) \circ \Lambda_{k} D u(x)\right|$ and so from (4.5) and (4.11),

$$
\left|\Lambda_{k} D v(x)\right| \leq\left|\Lambda_{k} D u(x)\right|
$$

for any $x \in \Omega$ and $k=1, \ldots, n$. Moreover, using (4.9), strict inequality holds in case $k=1$ if $u(x) \in \mathbb{R}^{N} \backslash B_{R}$ and $D u(x) \neq 0$. If $E \subset \subset \Omega$ is open and $|u(E) \backslash \mathcal{C}(u(\partial E))| \neq$ 0 , then by the area formula there exists $E^{\prime} \subset E$ with $\left|E^{\prime}\right| \neq 0,\left|u\left(E^{\prime}\right) \backslash \mathcal{C}(u(\partial E))\right| \neq 0$ and $|D u(x)| \neq 0$ for $x \in E^{\prime}$. By appropriate choice of $B_{R}$ it follows from (4.6) that $I[v]<I[u]$, contradicting the minimising property of $u$.

We next recall an application of the Courant-Lebesgue Lemma.

PROPOSITION 4.2. Suppose $u \in W^{1,2}\left(B_{R} ; \mathbb{R}^{2}\right)$. Then there exists a sequence $\rho_{n} \rightarrow$ 0 such that $\ell\left(u\left(\partial B_{\rho_{n}}\right)\right) \rightarrow 0$, where $\ell\left(u\left(\partial B_{\rho_{n}}\right)\right)$ is the length of $u\left(\partial B_{\rho_{n}}\right)$.

PROOF. First note that $\int_{\partial B_{\rho}}|D u|^{2}<\infty$ for a.e. $0<\rho<R$, and so $\left.u\right|_{\partial B_{\rho}}$ is continuous for a.e. $0<\rho<R$.

Let $M=\int_{B_{R}}|D u|^{2}$ and assume that $0<\delta<\sqrt{\delta}<R$. We claim that

$$
\int_{\partial B_{\rho}}\left|\frac{\partial u}{\partial \theta}\right|^{2} d \theta \leq \frac{2 M}{\log \frac{1}{\delta}}
$$

for some $\rho \in(\delta, \sqrt{\delta})$. 
Suppose not. Then

$$
\begin{aligned}
M & \geq \int_{B_{R}} \frac{1}{\rho^{2}}\left|\frac{\partial u}{\partial \theta}\right|^{2} \rho d \rho d \theta \\
& \geq \int_{\delta}^{\sqrt{\delta}} \frac{1}{\rho}\left(\int_{\partial B_{\rho}}\left|\frac{\partial u}{\partial \theta}\right|^{2} d \theta\right) d \rho \\
& >\frac{2 M}{\log \frac{1}{\delta}} \int_{\delta}^{\sqrt{\delta}} \frac{1}{\rho} d \rho \geq M .
\end{aligned}
$$

This contradiction establishes (4.14).

It follows that

$$
\begin{aligned}
\ell\left(u\left(\partial B_{\rho}\right)\right) & =\int_{\partial B_{\rho}}\left|\frac{\partial u}{\partial \theta}\right| d \theta \\
& \leq \sqrt{2 \pi}\left(\int_{\partial B_{\rho}}\left|\frac{\partial u}{\partial \theta}\right|^{2} d \theta\right)^{1 / 2} \\
& \leq \sqrt{\frac{4 \pi M}{\log \frac{1}{\delta}}}
\end{aligned}
$$

This establishes the Proposition.

Everywhere continuity of minimisers of (1.1) now follows from the previous two results.

THEOREM 4.3. Suppose $u \in W^{1,2}\left(\Omega ; \mathbb{R}^{2}\right)$ is a minimiser of (1.1). Then $u$ has a continuous representative.

PROOF. For each $x \in \Omega$ there exists by Proposition 4.2 a sequence $\rho_{n} \rightarrow 0$ such that $\ell\left(u\left(\partial B_{\rho_{n}}(x)\right)\right) \rightarrow 0$. By the maximum principle Theorem 4.1, $u\left(B_{\rho_{n}}(x)\right) \subset$ $\mathcal{C}\left(u\left(\partial B_{\rho_{n}}(x)\right)\right)$, and so diam $u\left(B_{\rho_{n}}(x)\right) \rightarrow 0$.

\section{Acknowledgement}

Most of this work was carried out while the first author was visiting the Centre for Mathematical Analysis at the Australian National University in Canberra. The support and the friendly atmosphere of the CMA is gratefully acknowledged. This research has been partially supported by the Australian Research Council. 


\section{References}

[1] E. Acerbi and N. Fusco, 'A regularity theorem for minimisers of quasi-convex integrals', Arch. Rational Mech. Anal. 99 (1981), 261-281.

[2] J. M. Ball, 'Constitutive inequalities and existence theorems in nonlinear elastostatics', in: Nonlinear analysis and mechanics: Heriot-Watt symposium, I (ed. R. J. Knops) (Pitman, London, 1977).

[3] - 'Convexity conditions and existence theorems in nonlinear elasticity', Arch. Rational Mech. Anal. 63 (1977), 337-403.

[4] L. C. Evans, 'Quasiconvexity and partial regularity in the calculus of variations', Arch. Rational Mech. Anal. 95 (1986), 227-252.

[5] L. C. Evans and R. F. Gariepy, 'Blow-up, compactness and partial regularity in the calculus of variations', Indiana Univ. Math. J. 36 (1987), 361-371.

[6] N. Fusco and J. E. Hutchinson, ' $C^{1, \alpha}$ partial regularity of functions minimising quasi-convex integrals', Manuscripta Math. 54 (1985), 121-143.

[7] — , 'Partial regularity in problems motivated by non-linear elasticity', SIAM J. Math. Anal. 22 (1991), 1516-1551.

[8] M. Giaquinta, 'Multiple integrals in the calculus of variations and nonlinear elliptic systems', volume 105 of Annals of Math. Studies (Princeton Univ. Press, Princeton, 1983).

[9] M. Giaquinta and G. Modica, 'Partial regularity of minimizers of quasi-convex integrals', Ann. Inst. H. Poincaré. Anal. Non Linéaire 3 (1986), 185-208.

[10] F. Leonetti, 'Maximum principles for functionals depending on minors of the Jacobian matrix of vector-valued mappings', Australian National University, preprint CMA-R20-1990.

[11] _ _ ' Maximum principles for vector-valued minimizers of some integral functionals', Boll. Un. Mat. Ital. A (6) 5 (1991), 51-56.

Dipartimento di Matematica "R. Caccioppoli"

Università di Napoli--Monte S. Angelo

Edificio T

via Cintia

80100 Napoli

Italy
Department of Mathematics School of Mathematical Sciences Australian National University

GPO Box 4

Canberra ACT 0200

Australia 